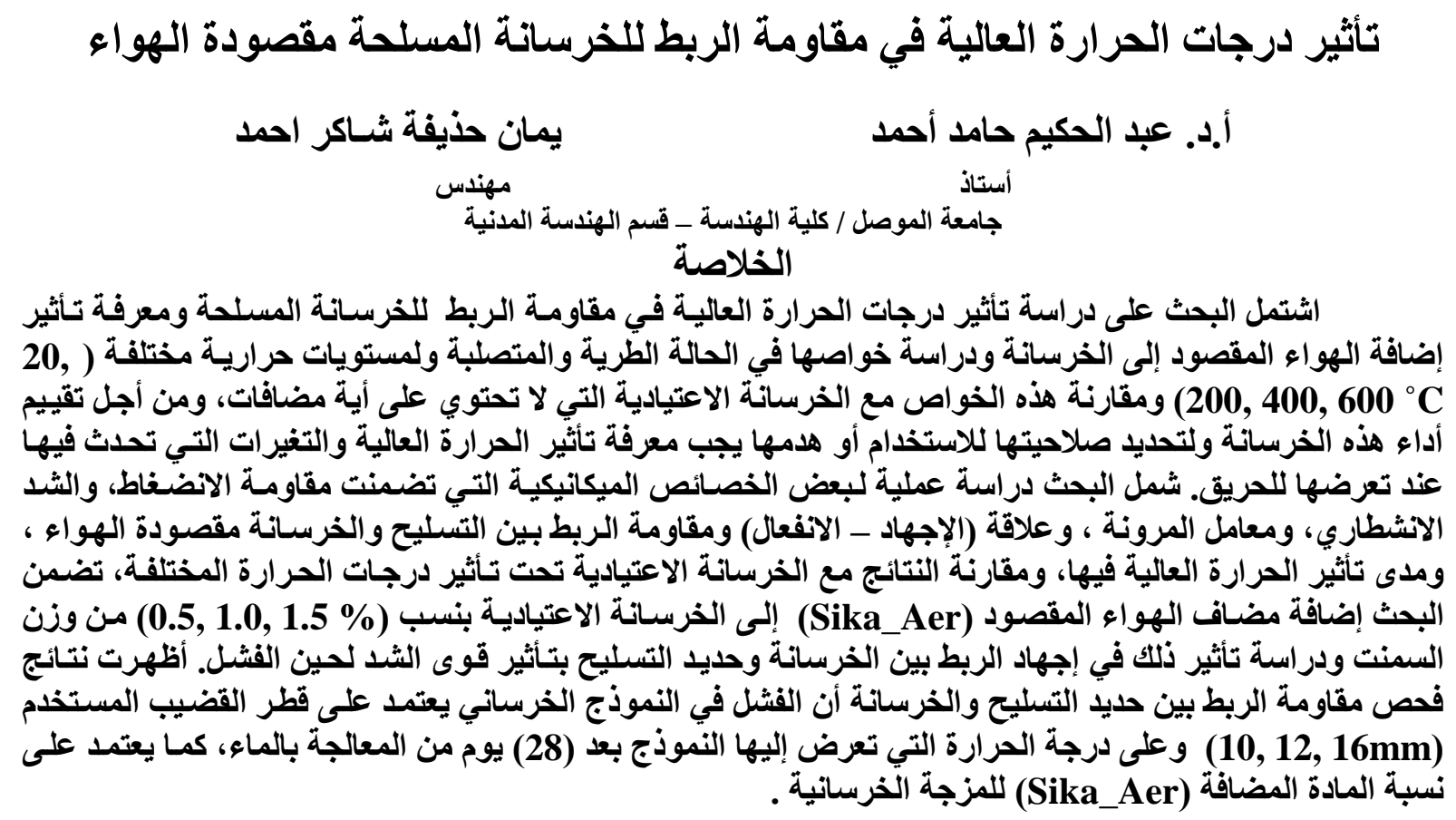

\title{
Effect of high temperature on Bond Strength in entrained air Reinforced Concrete
}

\author{
Dr. Abdul Hakeem Hamed Ahmed Yaman Huthaifa Shaker \\ Professor \\ Engineer
}

Civil Engrg. Dept. / Mosul Univ.

\section{Abstract}

This research aims to study the effect of temperature on bond strength in air entrained concrete and to find the effect of adding entrained air on the properties of the fresh and hardened Concrete under the influence of high temperatures of different levels $\left(20,200,400,600{ }^{\circ} \mathrm{C}\right)$. These properties are compared with those for normal concrete . In case of concrete structures exposed to an unexpected fire, changes are found in the properties of the concrete constituents, These changes are evaluated to assess the performance of these concrete structures and determine whether structures are safe or not .The research work includes an experimental study of the mechanical properties of the hardened concrete , and the effect of high temperature and entrained air admixture on concrete behavior including, compressive strength, splitting tensile strength, stress - strain relationship (modulus of elasticity) and bond strength between reinforcement and concrete. The work includes four different percentage of air entrained admixture (Sika_Aer) $(0,0.5,1.0,1.5 \%)$ by weight of cement. The effect of these variables on the bond stress between concrete and reinforcement as a result of tensile forces using pullout test are investigated. The Results show that the failure for reinforced concrete bond strength depends on diameter of the steel bar used $(\varnothing 10, \varnothing 12$ and $\emptyset 16 \mathrm{~mm})$, heating temperature for specimens after (28) days of water curing and also depends on the amount of admixture in concrete (Sika-Aer).

Keyword: Concrete, Bond Strength, Entrained air, High temperature

$$
\text { قبل: 2012-129 }
$$




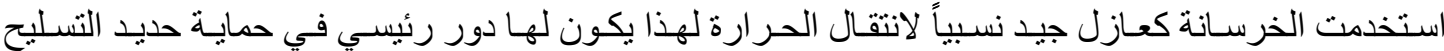

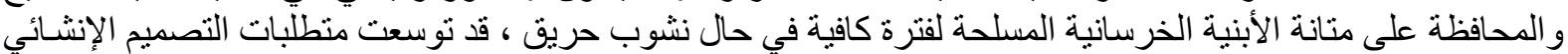

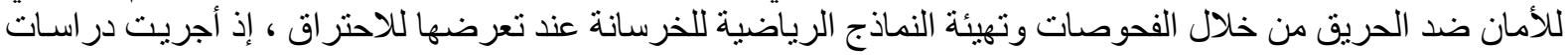

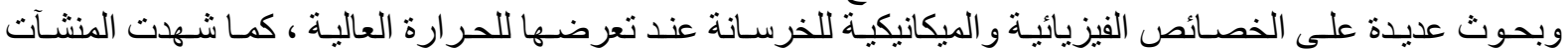

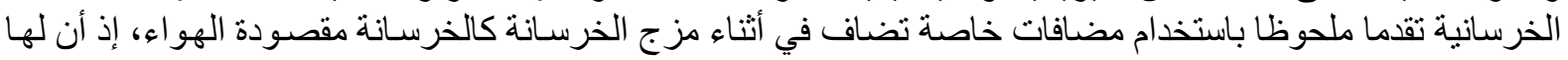

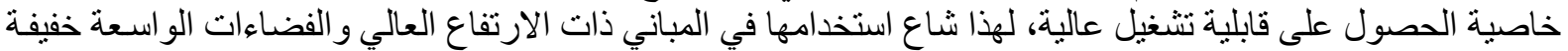

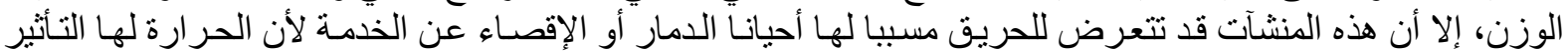

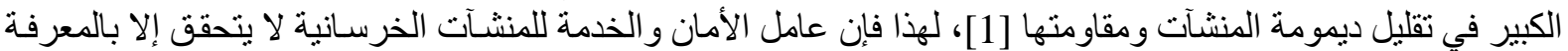

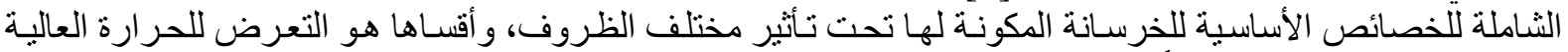
و التي تقود إلى الحريق - أحياناً - .

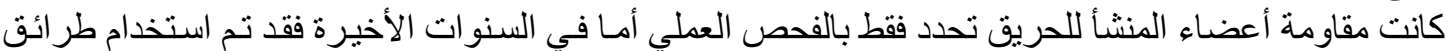

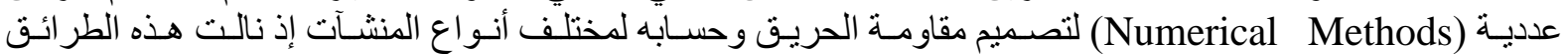

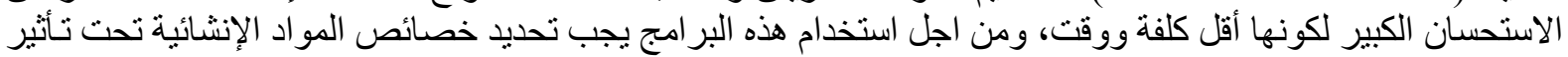

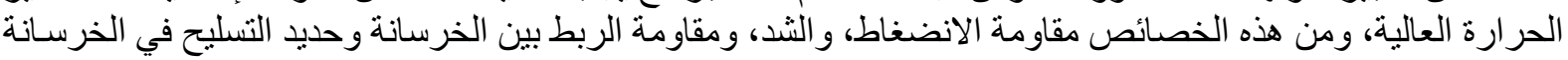

المسلحة [2].

مما تقدم في أعلاه تم اقتر اح هذا البحث لدر اسة تأثثر درجات الحرارة العالية في مقاومة الربط بين حديد التسليح

و الخرسانة مقصودة الهو اءو ومعرفة نوع أفع الفثل تحت إجهادات الربط.

2. 2 - 2 البرنامج العملي

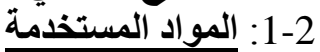

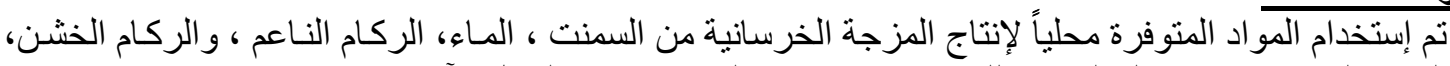

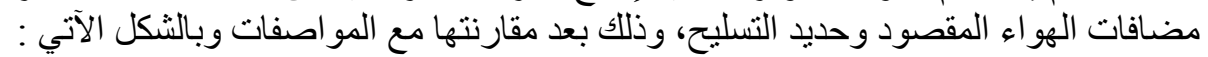

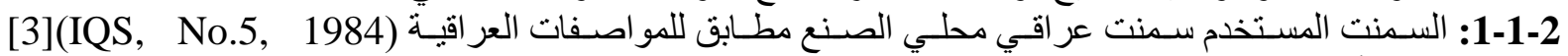
و المو اصفات الأمريكية (4STM C150-97) [4]، منتج في معمل سمنت بادوش. 2-1-2

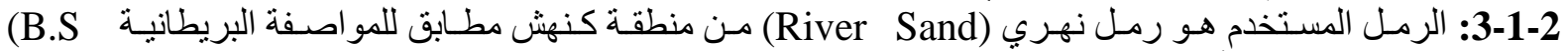

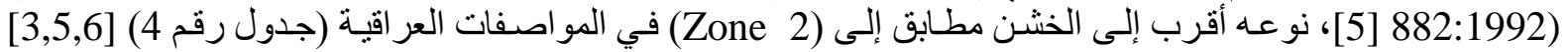

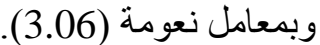

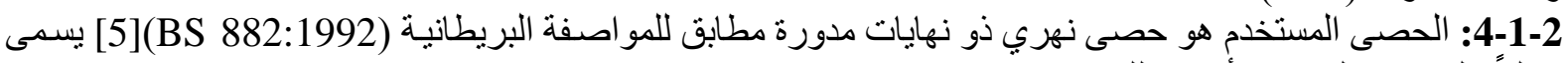

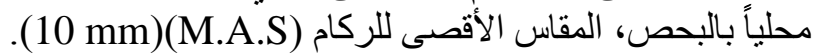

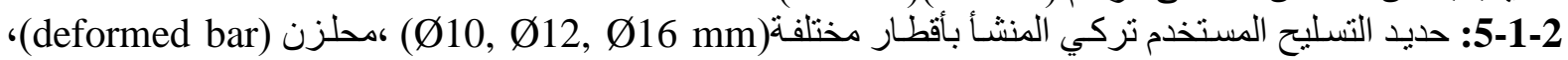

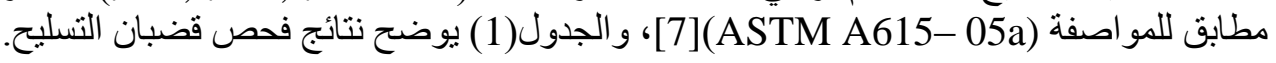

الجدول (1): نتائج فحص قضبان حديد التسليح

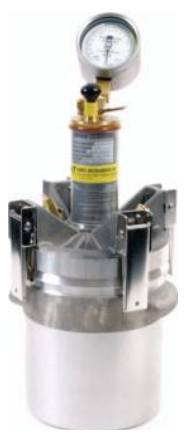

الثكل(1): الجهاز المستخدم

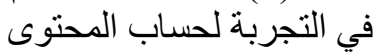
ألهو ائي للخرسانة الطرية

\begin{tabular}{|c|c|c|c|c|}
\hline & \multicolumn{2}{|c|}{ Yield } & \multicolumn{2}{c|}{ Ultimate } \\
\hline diameter & $\begin{array}{c}\text { Load } \\
(\mathrm{kN})\end{array}$ & $\begin{array}{c}\text { Stress } \\
(\mathrm{MPa})\end{array}$ & $\begin{array}{c}\text { Load } \\
(\mathrm{kN})\end{array}$ & $\begin{array}{c}\text { Strength } \\
(\mathrm{MPa})\end{array}$ \\
\hline $\boldsymbol{\varnothing 1 6} \mathbf{~ m m}$ & 97.19 & 483.38 & 128.77 & 640.45 \\
\hline$\varnothing 12 \mathbf{~ m m}$ & 65.41 & 578.35 & 78.08 & 690.38 \\
\hline$\varnothing 10 \mathbf{~ m m}$ & 44.45 & 565.955 & 53.41 & 680.03 \\
\hline
\end{tabular}

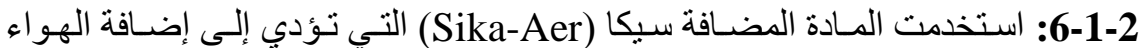

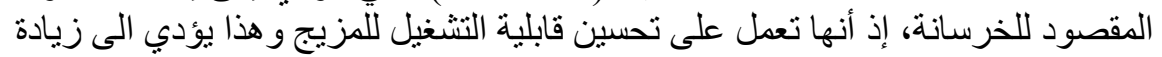

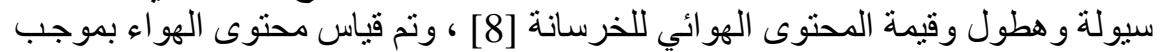

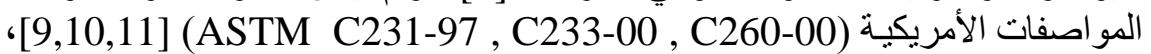
و الثكل (1) يوضح الجهاز المستخدم في التجربة . 
1-2-2

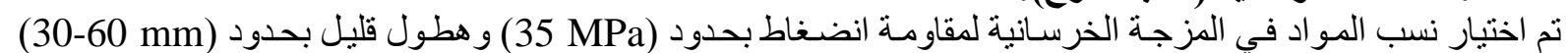

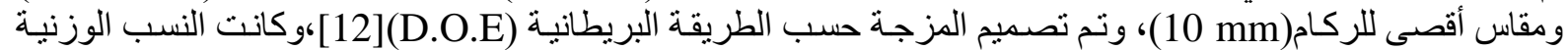

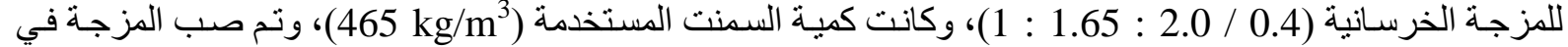
قو الب اسطو انية بأبعاد (150 x 300 mm) لنماذج فحوصات مقاومة الانضغاط ومعامل المرونة ومقاومـة الربط للتسليح

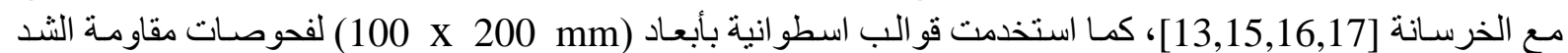

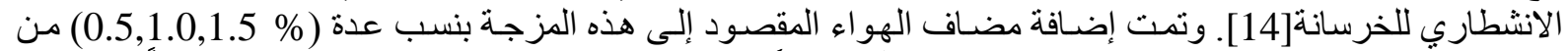

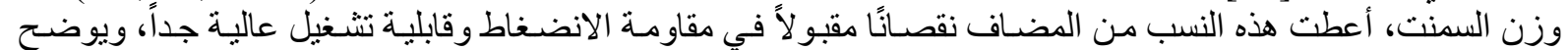
الجدول(2) نسب المادة المضافة للمزجة الخرن الخدانية .

\section{الجدول (2) : نسب المزجات الخرسانية}

\begin{tabular}{|c|c|c|c|}
\hline \% نسبة مضاف وزن الإسونت & ماء/ حصى النسب:رملية:سزنت للمزيج & المزجات الخرسانية & رقم المزجــة \\
\hline 0 & $1: 1.65: 2.0 / 0.4$ & مزجة اعتيادية & M1 \\
\hline 0.5 & $1: 1.65: 2.0 / 0.4$ & مقصودة الهو اء \% 0.5 سيكا & M2 \\
\hline 1.0 & $1: 1.65: 2.0 / 0.4$ & مقصودة الهو اء 1.0 سيكا & M3 \\
\hline 1.5 & $1: 1.65: 2.0 / 0.4$ & مقصودة الهو اء \% 1.5 سيكا & M4 \\
\hline
\end{tabular}

2-2-2

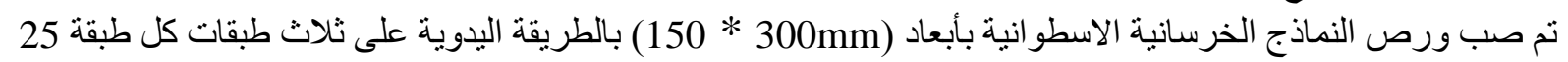

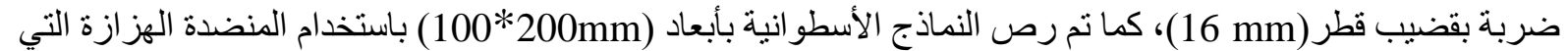

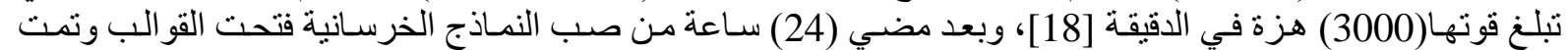

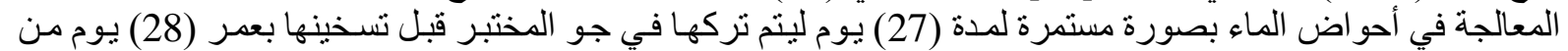

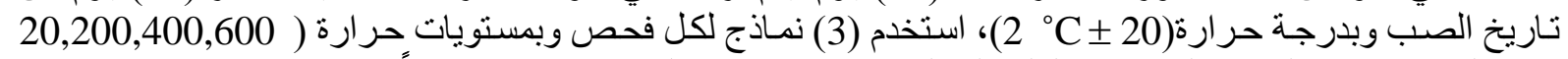

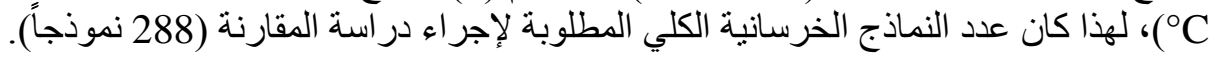

3-2: تسخين النماذج الخرسانية إلى درجات الحرارة العالية وتبريدها

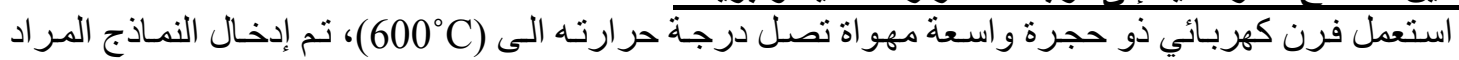

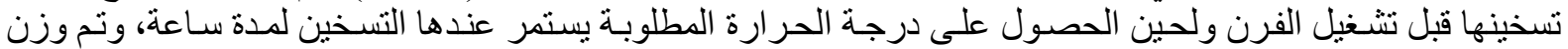

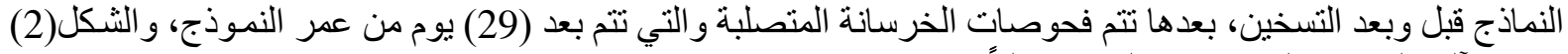

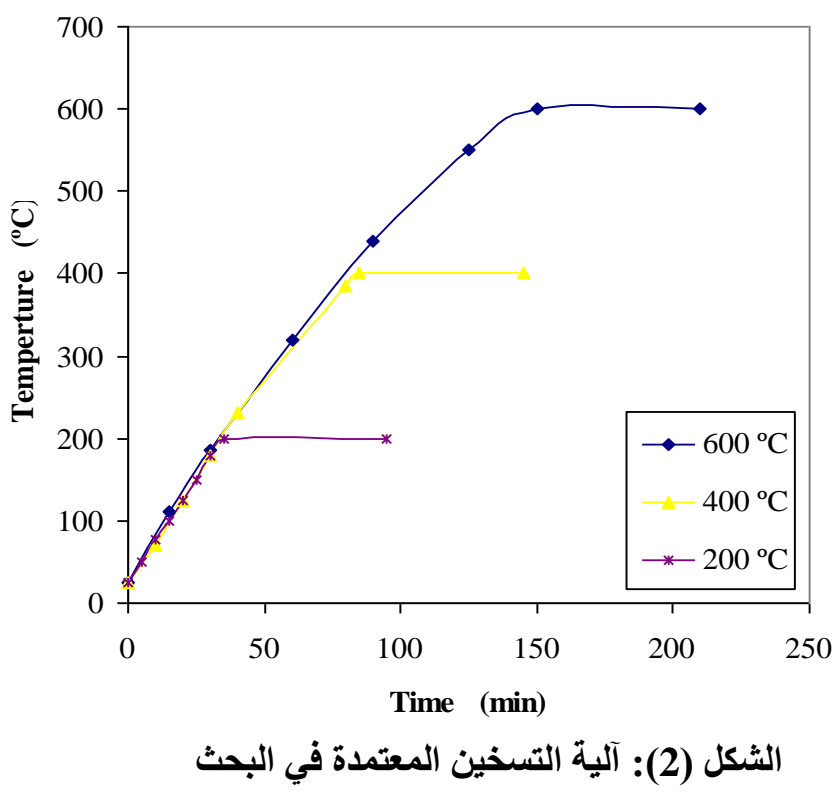
يوضح آلية التسخين المعتمدة في البحث عملياً.

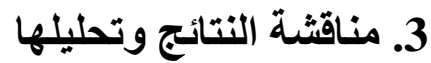

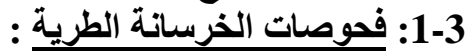

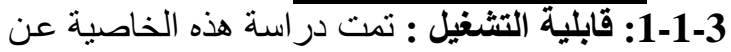

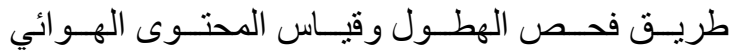

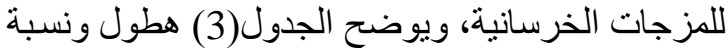
الهو اء للمزجات الخرسانية. 
الجدول (3): مقدار الهطول للمزجات الخرسانية ونسب الهواء داخلها .

\begin{tabular}{|c|c|c|}
\hline \multicolumn{2}{|c|}{$1: 1.65: 2.0 / 0.4$} & نسبة المزيج الخرساني \\
\hline 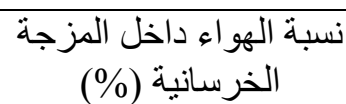 & $\begin{array}{c}\text { مقدار الهطول } \\
\text { (mm) }\end{array}$ & المزجات الخرسانية \\
\hline 2.0 & $5 \pm 65$ & المزجة الاعتيادية \\
\hline 4.25 & $5 \pm 115$ & المزجة مقصودة الهو اء بنسبة \% 0.5 سيكا \\
\hline 5.5 & $5 \pm 140$ & المزجة مقصودة الهو اء بنسبة \% 1.0 سيكا \\
\hline 6.5 & $5 \pm 180$ & المزجة مقصودة الهو اء بنسبة \% 1.5 سيكا \\
\hline
\end{tabular}

2-1-3: وقت التماسك الابتدائي : تم قياس وقت التماسك الابتدائي لعجينة السمنت وذللك باستعمال جهاز فايكت [19] ، وقد

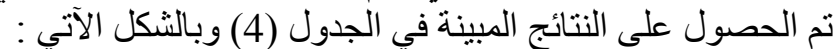

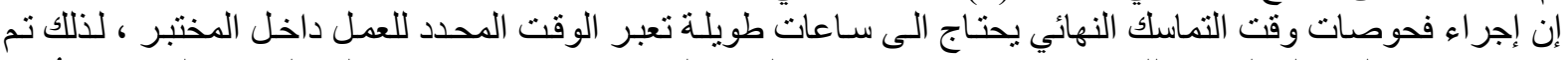

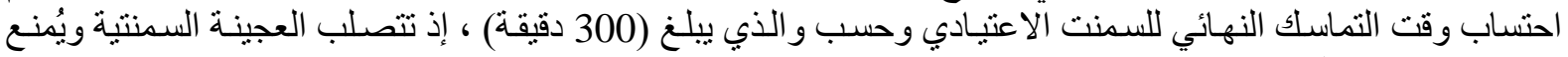

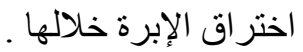

الجدول (4): وقت التماسك الابتدائي للعجينة الإسمنتية بمختلف أنواعها

\begin{tabular}{|c|c|}
\hline \multicolumn{2}{|c|}{ وقت التماسك الابتدائي للعجينة الإسمنتية بمختلف أنو اعها } \\
\hline وقت التماسك الابتدائي (min) & عجينة الاسمنت \\
\hline 90 & لمزجة الاعتيادية \\
\hline 85 & المزجة مقصودة الهواء بنسبة \% 0.5 سيكا \\
\hline 90 & لمزجة مقصودة الهواء بنسبة \% 1.0 سيكا \\
\hline 120 & المزجة مقصودة الهواء بنسبة \% 1.5 سيكا \\
\hline
\end{tabular}

2-3 : فحوصات الخرسانة المتصلبة:

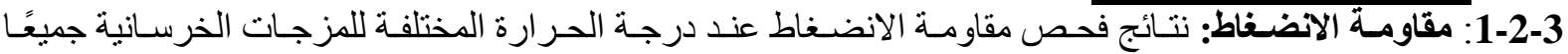

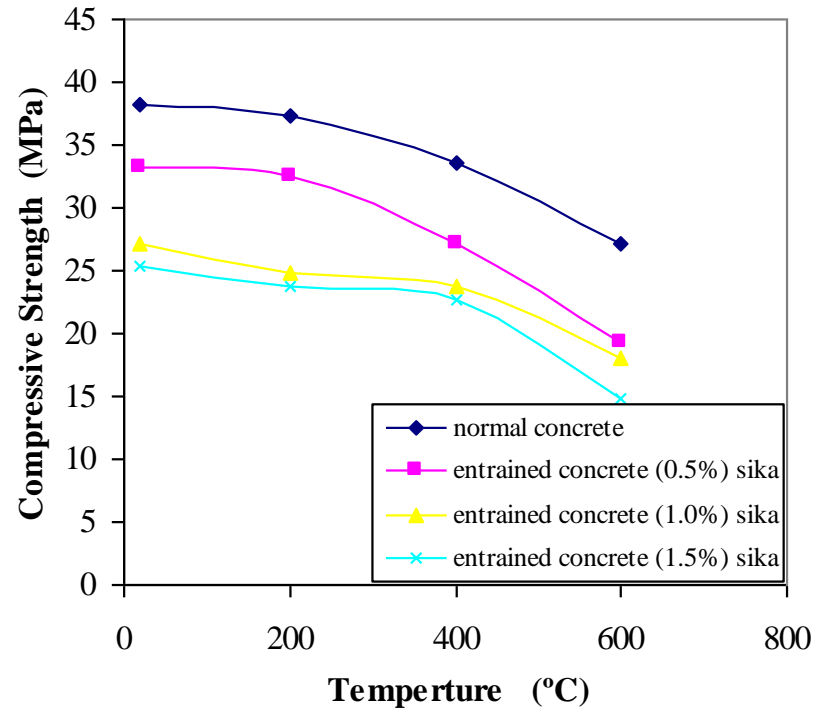

الثكل(3): مقاومة انضغاط المزجات الخرسانية تحت تأثير درجات الحرارة العالية

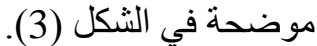

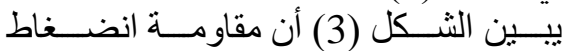

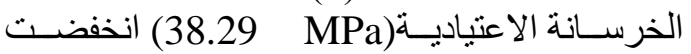
بنسبة(13.4, 29.3, 33.6 بعد إضـافة مضـاف

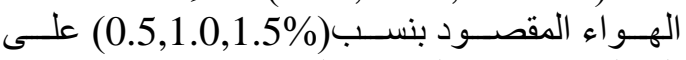

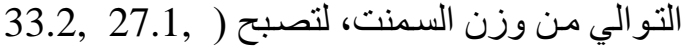
(25.4 MPa

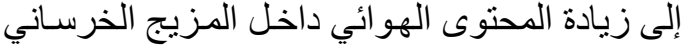

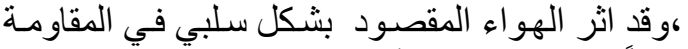

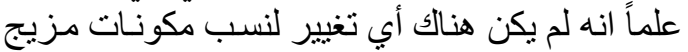
الخرسانة الاعتيادية كنسب الركام و السمنت أو المين المـاء

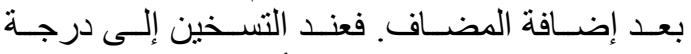

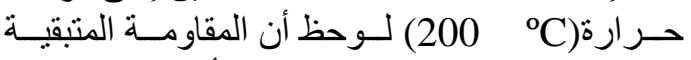

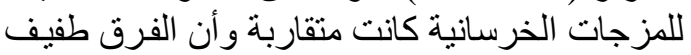

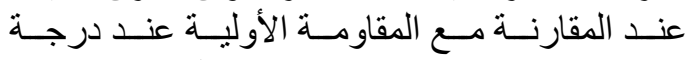

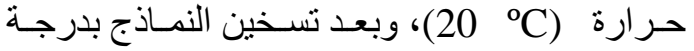

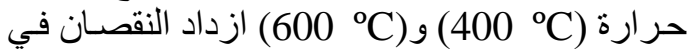

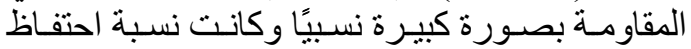

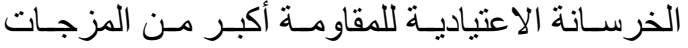




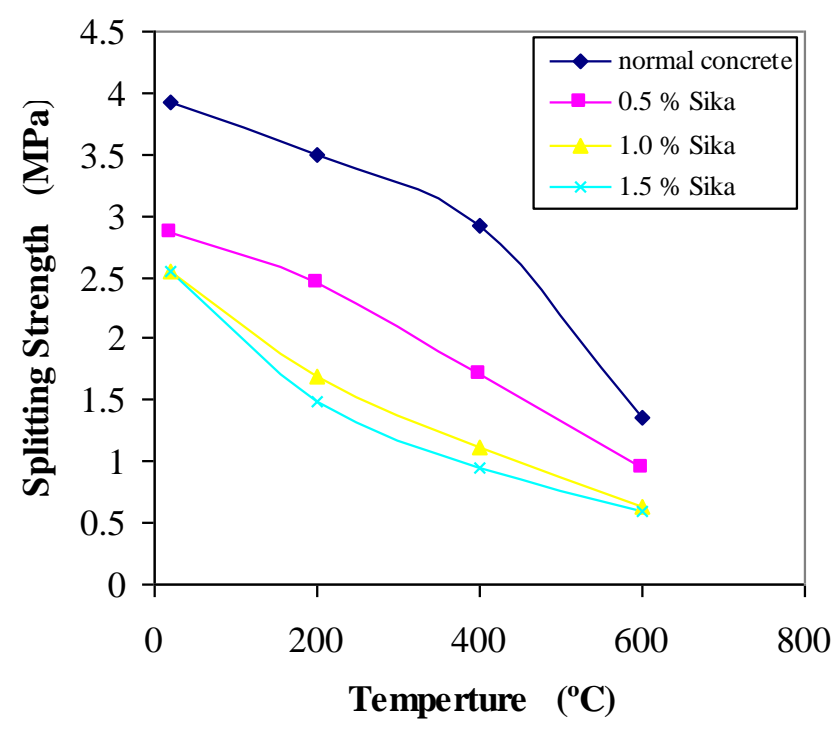

الثكل(4): مقاومة الثد الانشطاري للمزجات الخرسانية تحت تأثير درجات الحرارة العالية

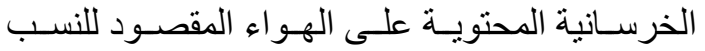

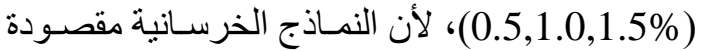

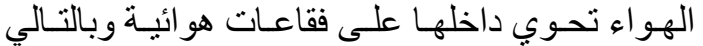

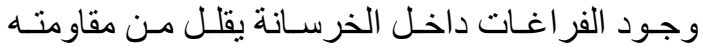

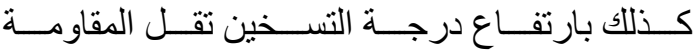
.[1,2,12,20]

2-2-3: مقاومسة الثـــ الانشـطاري: تتـأثر بالعو امـل

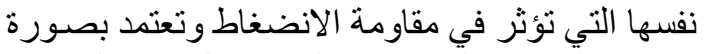

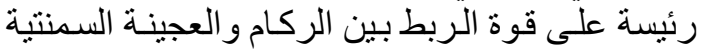

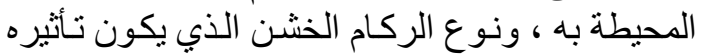

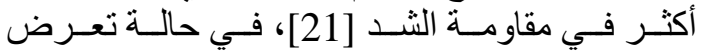

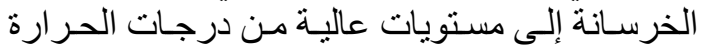

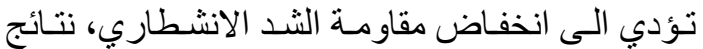

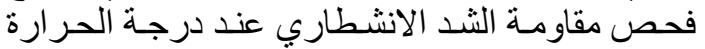

المختلفة للمزجات جميعًا موضحة في الثكل فئل (4).

يبين الثكل (4) أن مقاومة الثد الانشطاري

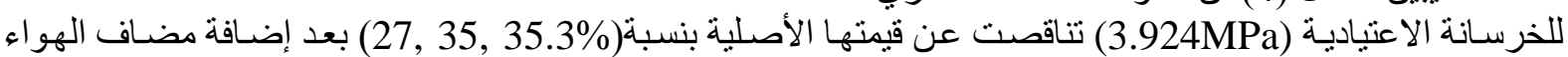

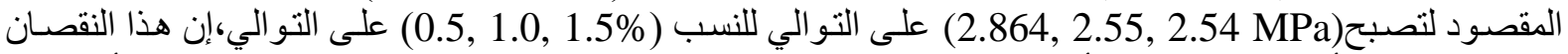

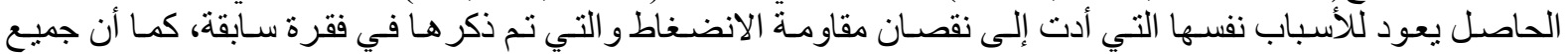

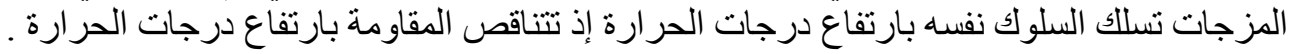

3-3-2-3: معامل المرونة: يعتمد على مقاومة الخرسانة وينأثر بنسب الركام وصلابته، وعمر الخرسانة، وظروف الفحص،

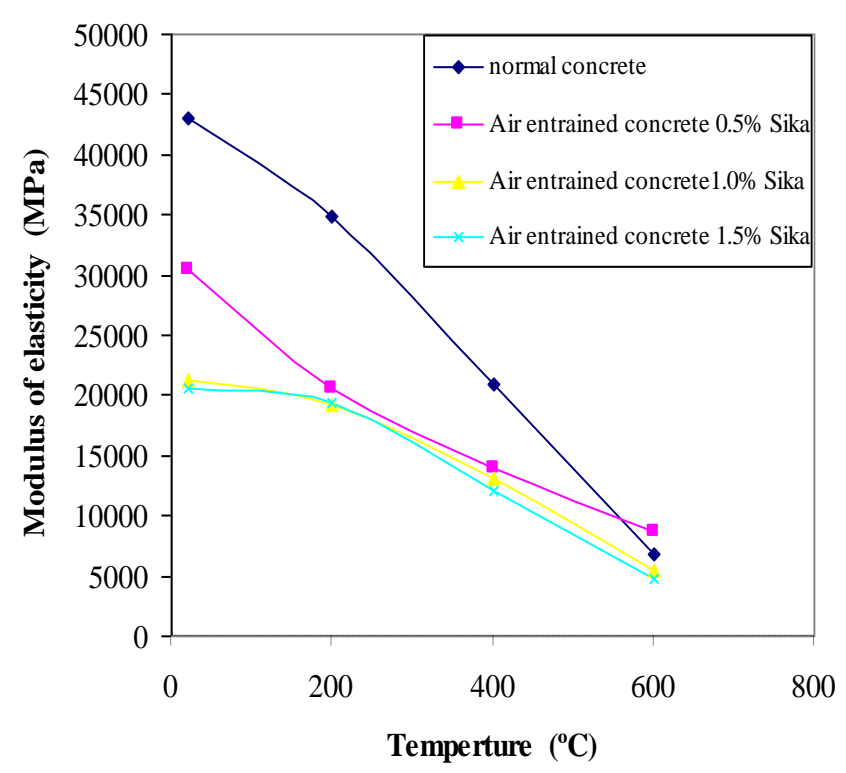

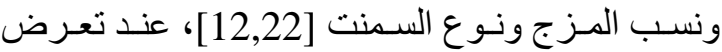

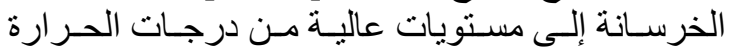

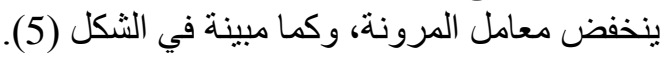

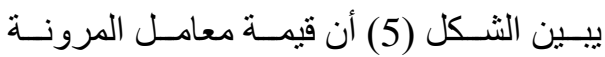

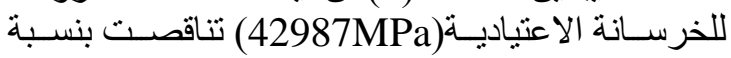

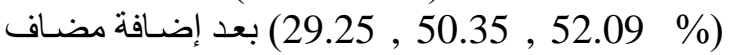

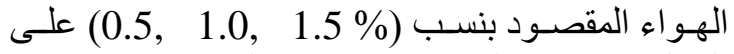

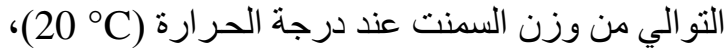

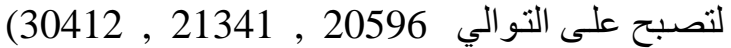
MPa)

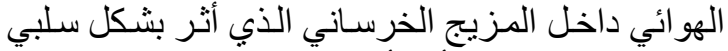

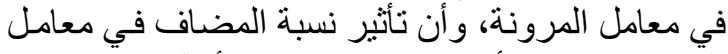

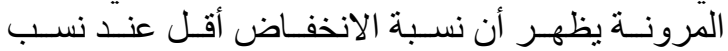
الإضافة (1.0,1.5\%).

ون-2-3 مقاومة الربط بين حديد التسليح والخرسـانة:

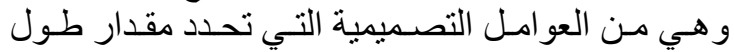

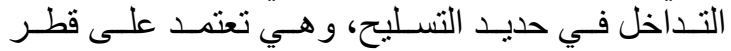

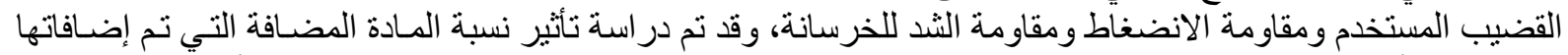

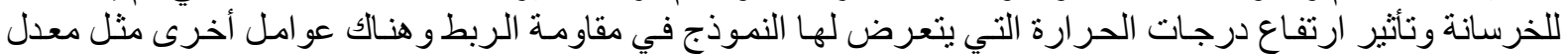

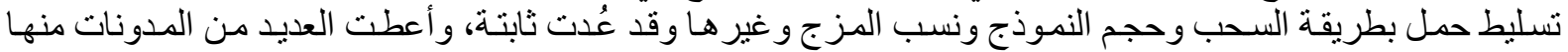

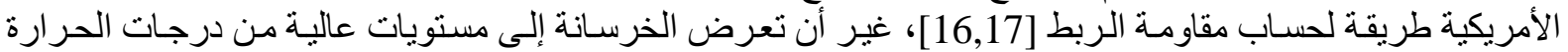

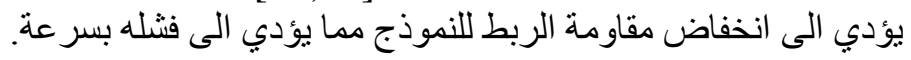


الجدول (5) : الحمل ، الإزاحة ، الإجهاد والانفعال القصوى للمزجات الخرسانية كافة ، وللارجات الحرارية وللأقطار المستخدمة كافة لحديد التسليح

\begin{tabular}{|c|c|c|c|c|c|c|c|c|}
\hline Temperature & Diameter & Mixtures & $\begin{array}{l}\text { Max. } \\
\text { Load }\end{array}$ & $\begin{array}{l}\text { Max. } \\
\text { lower }\end{array}$ & $\begin{array}{l}\text { Max. } \\
\text { upper }\end{array}$ & subtract & $\begin{array}{c}\text { Max. } \\
\text { Strain }\end{array}$ & $\begin{array}{c}\text { Max. } \\
\text { Stress }\end{array}$ \\
\hline \multirow[t]{6}{*}{$\left({ }^{\circ} \mathbf{C}\right)$} & $(\mathbf{m m})$ & & $(\mathbf{k N})$ & $(\mathbf{m m})$ & $(\mathbf{m m})$ & $(\mathbf{m m})$ & $(\mathbf{m m} / \mathbf{m m})$ & (MPa) \\
\hline & $\emptyset 16 \mathrm{~mm}$ & Normal & 110 & 7.1 & 1.2 & 5.9 & 0.0196 & 7.295 \\
\hline & & 0.5\% Sika & 85.5 & 1.22 & 1.05 & 0.17 & 0.000566 & 5.67 \\
\hline & & $1 \%$ Sika & 60.5 & 0.92 & 0.76 & 0.16 & 0.00053 & 4.012 \\
\hline & & 1.5\% Sika & 90 & 2.24 & 0.53 & 1.71 & 0.0057 & 5.97 \\
\hline & $\emptyset 12 \mathrm{~mm}$ & Normal & 77.5 & 10.37 & 0.72 & 9.65 & 0.0321 & 6.852 \\
\hline \multirow[t]{12}{*}{$20{ }^{\circ} \mathrm{C}$} & & 0.5\% Sika & 72 & 9.2 & 1.22 & 7.98 & 0.0266 & 6.366 \\
\hline & & $1 \%$ Sika & 61 & 2 & 1.6 & 0.4 & 0.00133 & 5.393 \\
\hline & & $1.5 \%$ Sika & 73 & 9 & 0.83 & 8.17 & 0.0272 & 6.454 \\
\hline & $\emptyset 10 \mathrm{~mm}$ & Normal & 52 & 7 & 0.45 & 6.55 & 0.0218 & 5.517 \\
\hline & & 0.5\% Sika & 49.5 & 5.87 & 1.46 & 4.41 & 0.0147 & 5.252 \\
\hline & & $1 \%$ Sika & 41.5 & 2.18 & 1.72 & 0.46 & 0.00153 & 4.403 \\
\hline & & $1.5 \%$ Sika & 51 & 6 & 1.4 & 4.6 & 0.0153 & 5.411 \\
\hline & $\emptyset 16 \mathrm{~mm}$ & Normal & 123 & 13.5 & 0.76 & 12.74 & 0.04246 & 8.156 \\
\hline & & 0.5\% Sika & 57.5 & 1.2 & 0.86 & 0.34 & 0.00113 & 3.813 \\
\hline & & $1 \%$ Sika & 51 & 0.84 & 0.68 & 0.16 & 0.000533 & 3.382 \\
\hline & & $1.5 \%$ Sika & 50 & 1.1 & 0.4 & 0.7 & 0.00233 & 3.316 \\
\hline & $\emptyset 12 \mathrm{~mm}$ & Normal & 75 & 8.17 & 0.46 & 7.71 & 0.0257 & 6.631 \\
\hline \multirow[t]{12}{*}{$200{ }^{\circ} \mathrm{C}$} & & 0.5\% Sika & 56 & 1.87 & 1.46 & 0.41 & 0.00136 & 4.951 \\
\hline & & $1 \%$ Sika & 51.5 & 1.2 & 0.78 & 0.42 & 0.0014 & 4.553 \\
\hline & & 1.5\% Sika & 60 & 1.66 & 0.83 & 0.83 & 0.00276 & 5.305 \\
\hline & $\emptyset 10 \mathrm{~mm}$ & Normal & 52 & 10.24 & 0.4 & 9.84 & 0.0328 & 5.517 \\
\hline & & 0.5\% Sika & 45.5 & 6 & 2.2 & 3.8 & 0.0126 & 4.827 \\
\hline & & $1 \%$ Sika & 41.5 & 1.38 & 1.14 & 0.24 & 0.0008 & 4.403 \\
\hline & & $1.5 \%$ Sika & 47.5 & 2.5 & 1.3 & 1.2 & 0.004 & 5.04 \\
\hline & $\emptyset 16 \mathrm{~mm}$ & Normal & 115 & 11.64 & 0.75 & 10.89 & 0.0363 & 7.626 \\
\hline & & 0.5\% Sika & 55 & 1.3 & 1.09 & 0.21 & 0.0007 & 3.647 \\
\hline & & $1 \%$ Sika & 60 & 1.1 & 0.7 & 0.4 & 0.00133 & 3.98 \\
\hline & & 1.5\% Sika & 65 & 1.2 & 0.69 & 0.51 & 0.0017 & 4.31 \\
\hline & $\emptyset 12 \mathrm{~mm}$ & Normal & 75 & 7.24 & 0.4 & 6.84 & 0.0228 & 6.631 \\
\hline \multirow[t]{12}{*}{$400{ }^{\circ} \mathrm{C}$} & & 0.5\% Sika & 65 & 3.98 & 2.03 & 1.95 & 0.0065 & 5.747 \\
\hline & & $1 \%$ Sika & 57 & 1.72 & 0.8 & 0.92 & 0.00306 & 5.04 \\
\hline & & $1.5 \%$ Sika & 67.5 & 4 & 1.7 & 2.3 & 0.00766 & 5.97 \\
\hline & $\emptyset 10 \mathrm{~mm}$ & Normal & 52 & 11 & 0.44 & 10.56 & 0.0352 & 5.517 \\
\hline & & 0.5\% Sika & 51.5 & 5.6 & 0.9 & 4.7 & 0.01566 & 5.411 \\
\hline & & $1 \%$ Sika & 48 & 3 & 0.85 & 2.15 & 0.00716 & 5.093 \\
\hline & & $1.5 \%$ Sika & 47.5 & 4 & 1.5 & 2.5 & 0.00833 & 5.04 \\
\hline & $\emptyset 16 \mathrm{~mm}$ & Normal & 90 & 0.83 & 0.7 & 0.13 & 0.000433 & 5.968 \\
\hline & & 0.5\% Sika & 40 & 1.6 & 1.16 & 0.44 & 0.00146 & 2.652 \\
\hline & & $1 \%$ Sika & 46 & 1.11 & 0.82 & 0.29 & 0.000966 & 3.05 \\
\hline & & 1.5\% Sika & 37.5 & 1.34 & 0.85 & 0.49 & 0.00163 & 2.487 \\
\hline & $\emptyset 12 \mathrm{~mm}$ & Normal & 74 & 3.4 & 0.47 & 2.93 & 0.00976 & 6.543 \\
\hline \multirow[t]{7}{*}{$600{ }^{\circ} \mathrm{C}$} & & 0.5\% Sika & 35.5 & 1.33 & 0.95 & 0.38 & 0.00126 & 3.139 \\
\hline & & $1 \%$ Sika & 41 & 1.62 & 0.9 & 0.72 & 0.0024 & 3.625 \\
\hline & & $1.5 \%$ Sika & 42 & 1.4 & 0.95 & 0.45 & 0.0015 & 3.713 \\
\hline & $\emptyset 10 \mathrm{~mm}$ & Normal & 51.5 & 12 & 0.54 & 11.46 & 0.0382 & 5.464 \\
\hline & & 0.5\% Sika & 25 & 2.38 & 2.1 & 0.28 & 0.000933 & 2.652 \\
\hline & & $1 \%$ Sika & 29 & 1.25 & 1 & 0.25 & 0.000833 & 3.077 \\
\hline & & $1.5 \%$ Sika & 30 & 1.4 & 1.17 & 0.23 & 0.000766 & 3.183 \\
\hline
\end{tabular}




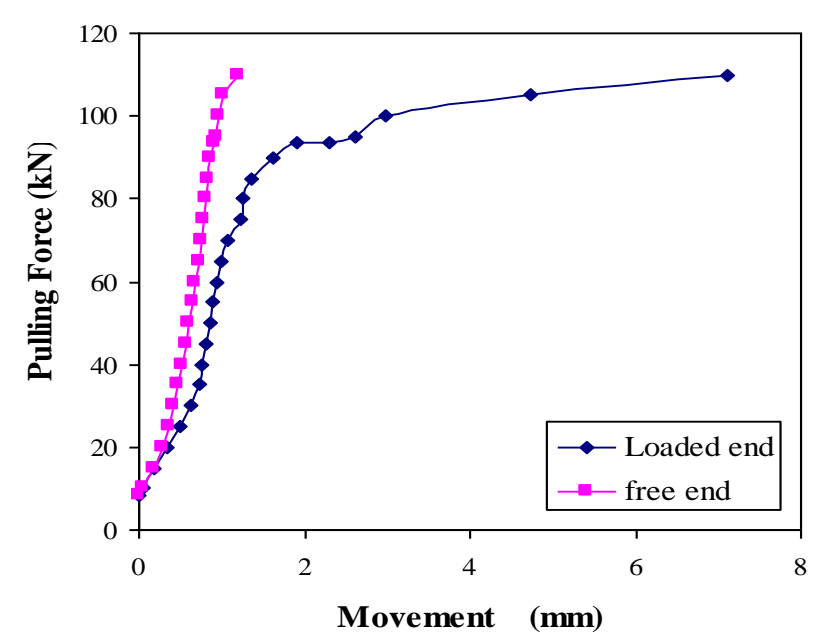

الشكل (6): علاقات (الحمل - الإزاحة) للخرسانة

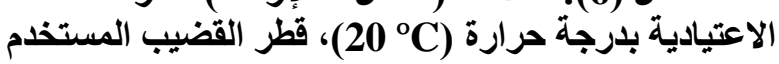

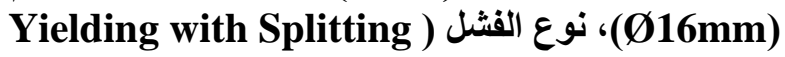
failure)

ويبين الثـكل (6) مثنال عن سـلوك النمـاذج الخاصـة

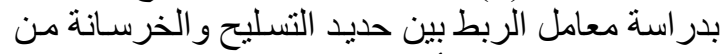

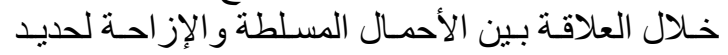

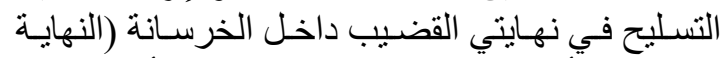

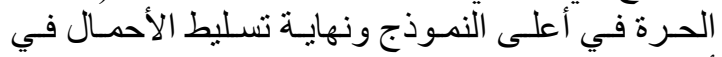
أسفل النموذج).

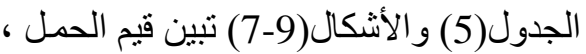

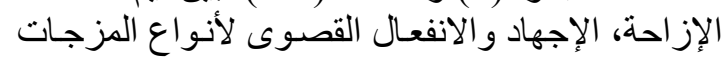

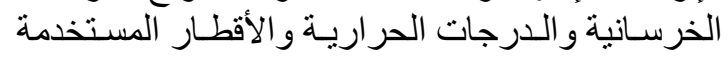
لحديد التسليح.

5-2-3

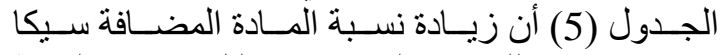

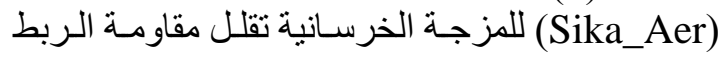

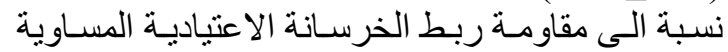
0.5, 1.0, (7.295 MPa) 77.7, ) 1.5\%

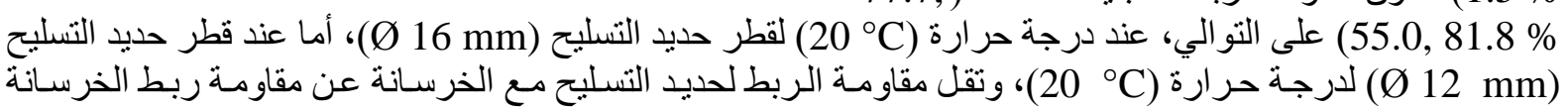

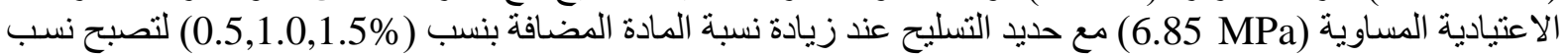

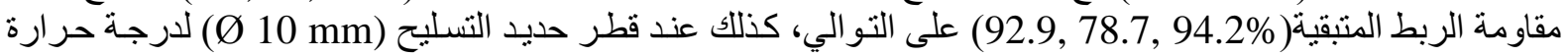

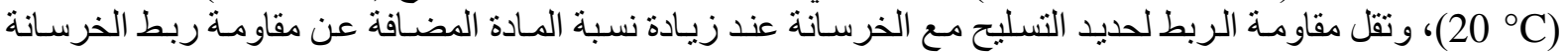

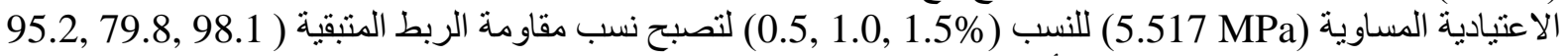

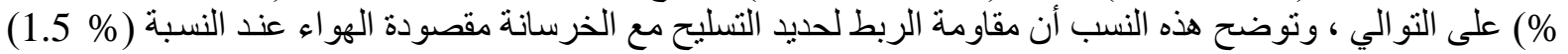

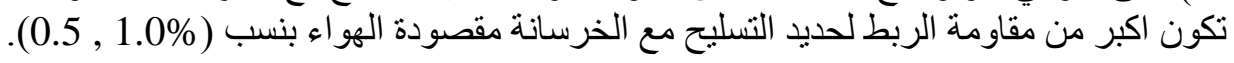

5-2-3

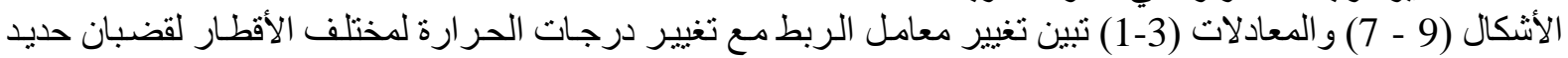

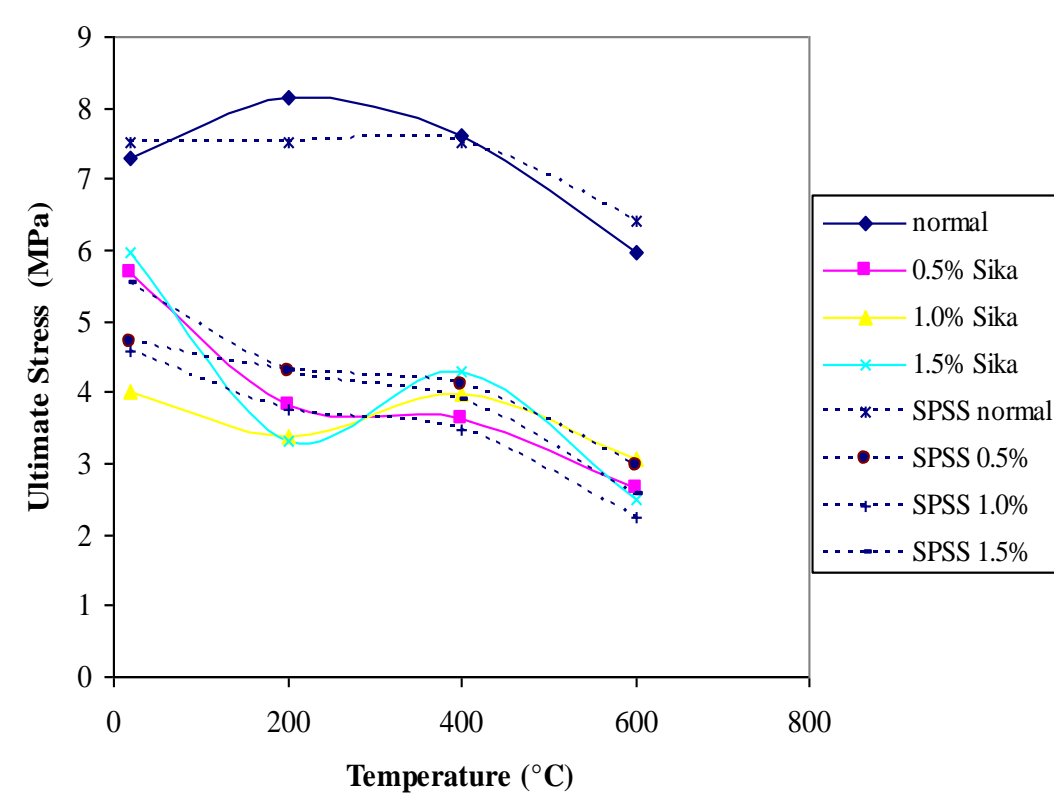

التسـليح (10, 12 (10)

ولنسب مختلفة من المضـاف (16, ) . $(0.5,1.0,1.5 \%$

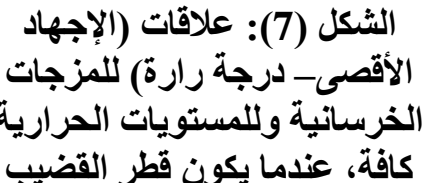

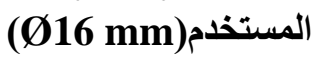

$$
\sigma=\left(-19.9 \times \mathrm{T}^{-0.017}\right) \times(\mathrm{M})-\left(2.74 \times \mathrm{T}^{8.86}\right)+\left(586.94 \times \mathrm{M}^{1.007}\right)-(568.95 \times M)+7.533
$$

$$
\left(\mathbf{R}^{2}=\mathbf{0 . 9 0 2}\right)
$$



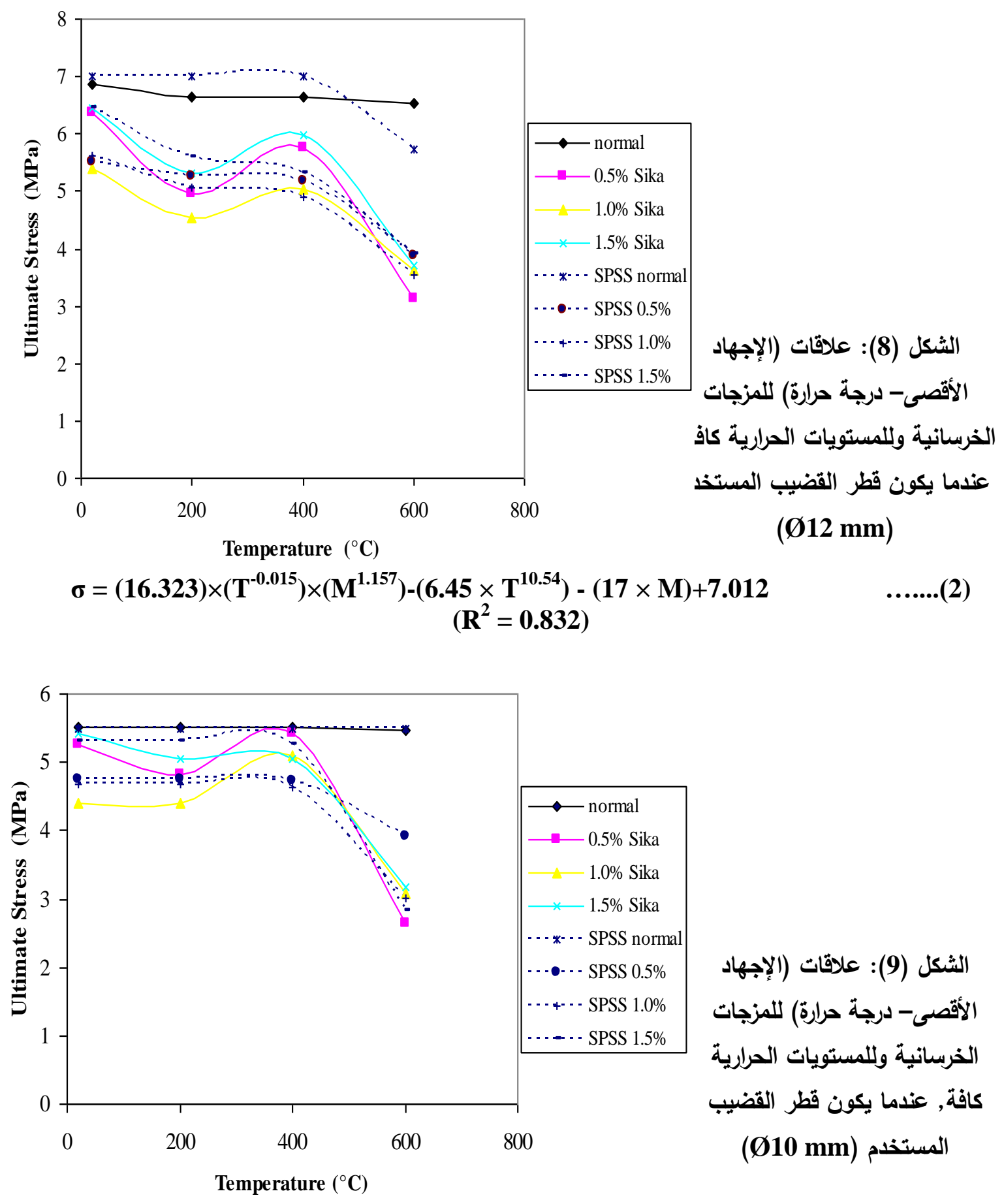

الشكل (9): علاقات (الإجهاد

الأقصى - درجة حرارة) للمزجات

الخرسانية وللمستويات الحرارية

كافة, عندما يكون قطر القضيب

المستخدم (Ø10 mm)

$\sigma=(-4.74) \times\left(T^{9.55}\right) \times(M)+\left(1.29 \times M^{2.07}\right)-(2.13 \times M)+5.504$

T : درجة حرارة التسخين ( T

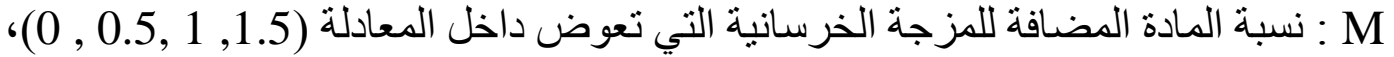

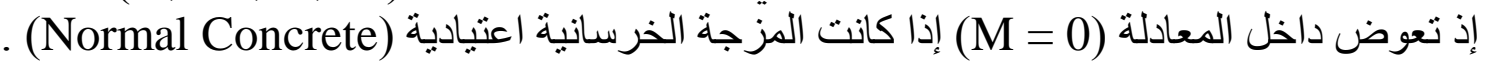

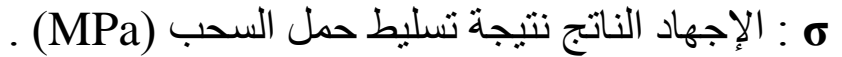


يُلاحظ من الأشكال (9 - 7) أن سلوك المزجات الخرسانية مقصودة الهو اء عند أقصى مقدار للإجهاد والانفعال

للنسب المضـافة الثثلاث (0.5 \%

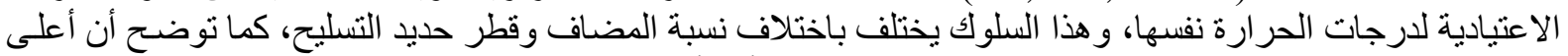

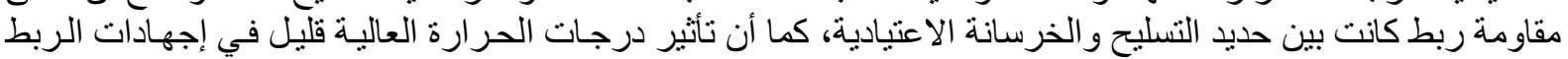

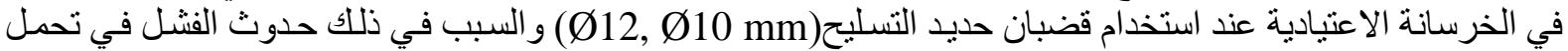

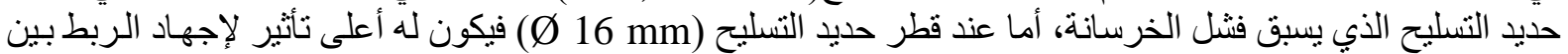

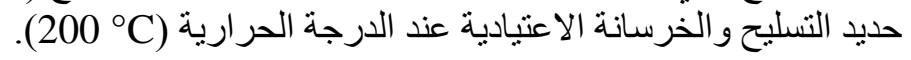

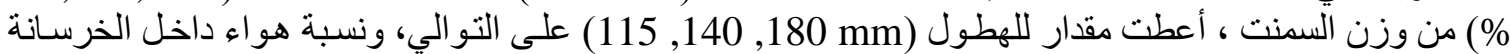

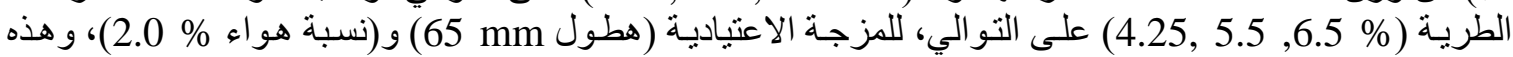

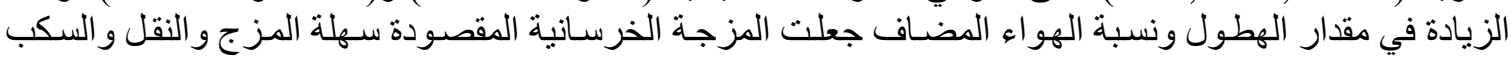

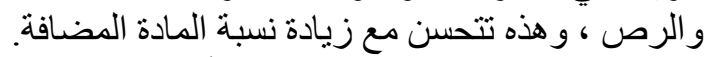

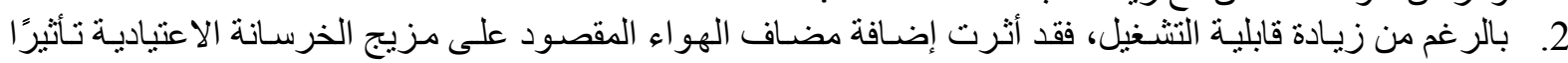

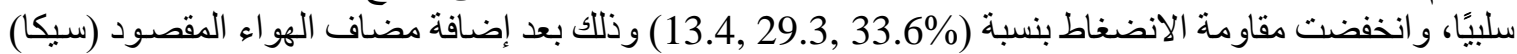
بنسب (1.5 1.0 1.0, 0.5) على التو الي من وزن السمنت عن مقاومة الانضغاط الأصلية قبل الإضافة ، و هذا التأتئير

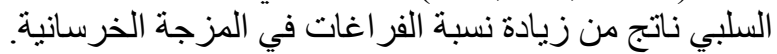

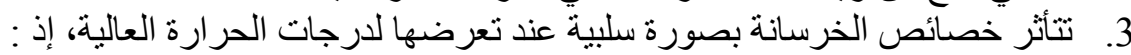

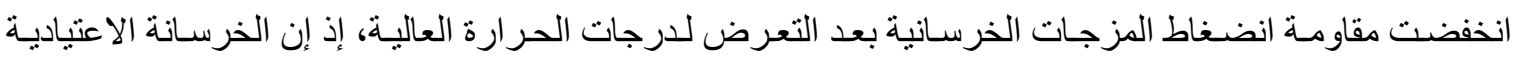

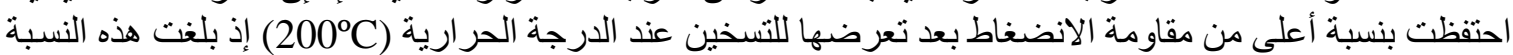
. $(97.53 \%)$

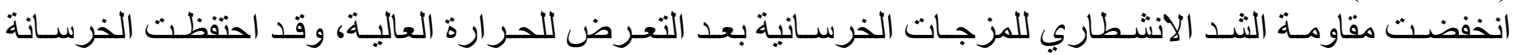

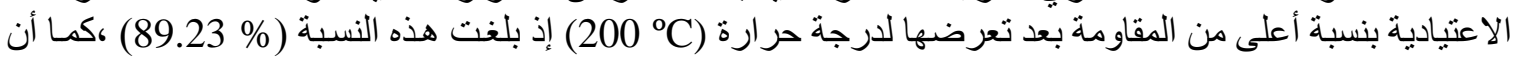
مقاومة الثد الانشطاري للخرسانة الاعتيادية عند درجة حرارة (3.

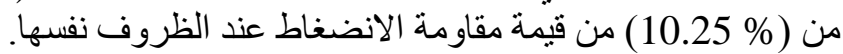

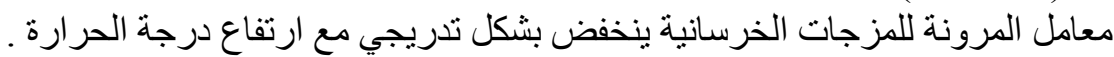

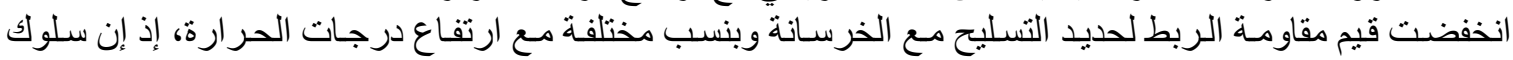

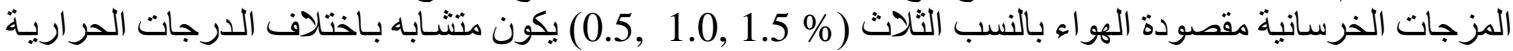

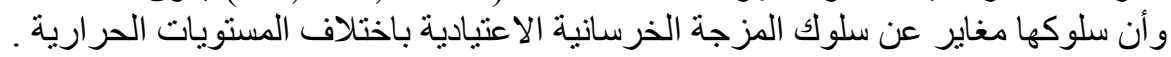

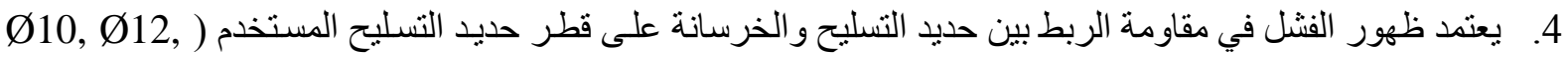
له mm نسبة المادة المضافة للمزجة.

[1] Husem, M ., " The Effect of High Temperature on Compressive and Flexural Strengths of Ordinary and High performance Concrete " , Fire Safety Journal , Vol. 41 , 2006 , pp. 155163 .

[2] Cheng, F.P. , Kodur V.K.R. and Wang T.C. ," Stress- Strain Curves for High Strength Concrete at Elevated Temperatures" ,Journal of Materials in Civil Engineering ,Vol.16 ,No.1 , February, 2004, pp. 84-94.

[3] المواصففة القياسـية العر اقيـة (رقم 5) ،" خصـائص السـنت البورتلنـدي الاعتبـادي " ، الجهـاز المركزي للتقيبس و السيطرة النوعية ، 1984 .

[4] ASTM C150-97,"Standard Specification for Portland Cement", American Society for Testing and Materials . 
[5] BS 882 - 1992 ,"Aggregate from Natural Source for Concrete " , British Standard Institution , 1992 .

[6] المو اصفة القياسية العر اقية (رقم 45) ، جدول رقم (4)،" ركام المصادر الطبيعية المستعمل في الخرسـانة والبناء" ،

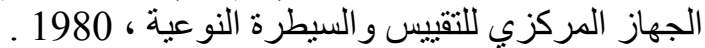

[7] ASTM A615/615M-05a ," Standard Specification for Deformed and Plain Billet-Steel Bars for Concrete Reinforcement ", American Society for Testing and Materials .

[8] Hamad, B.S. , Rteil, A.A. , EL- Fadel ,M. , " Effect of Used Engine Oil on Properties of Fresh and Hardened Concrete ", Construction and Building Materials (Elsevier), Vol. 17 ,Issue 5 , July , 2003 , pp.311-318 .

[9] ASTM C231-99 , " Standard Test Method for Air Content of Freshly Mixed Concrete by the Pressure Method ", American Society for Testing and Materials .

[10] ASTM C233-01 ,"Standard Test Method for Air-Entraining Admixture for Concrete ", American Society for Testing and Materials .

[11] ASTM C260-01 ,"Standard Specification for Air-Entraining Admixture for Concrete ", American Society for Testing and Materials .

[12] Neville , A. M. (2002), Properties of Concrete , 3rd Edition , Pitman Publishing Ltd, London .

[13] ASTM C39-04,"Compressive Strength of Cylindrical Concrete Specimens ", American Society for Testing and Materials .

[14] ASTM C496-04," Splitting Tensile Strength of Cylindrical Concrete Specimens", American Society for Testing and Materials.

[15] ASTM C469-02 ," Elastic Modulus of Elasticity and Poisson's Ratio of Concrete in Compression",American Society for Testing and Materials

[16] ASTM C234-69," Standard Test Method for Comparing Concretes on the Basis of the Bond Developed with Reinforcing Steel ", American Society for Testing and Materials .

[17] ASTM A944-99 ,"Standard Test Method for Comparing Bond Strength of Steel Reinforcing Bars to Concrete Using Beam-End Specimens ", American Society for Testing and Materials .

[18] ASTM C192-02,"Standard Practice for Making and Curing Concrete Test Specimens in the Laboratory", American Society for Testing and Materials .

[19] ASTM C191-99 ,"Standard Test Method for Time of Setting of Hydraulic Cement by Vicat Needle", American Society for Testing and Materials .

[20] Skokie, IL. ," Procedures for Evaluating Air Entraining Admixtures for Highway Concrete ", Construction Technology Laboratories, Inc. , National Cooperative Highway Research Program , January, 2006.

[21] ACI Committee 318-05 ," Building Code Requirements for Structural Concrete ACI", American Concrete Institute , Farmington Hills , Michigan , 2005.

[22] Troxell ,G.E. , Davis ,H.E. and Killy , J.W. , "Composition and Properties of Concrete " , 2nd Edition , 1965 .

تم اجراء البحث في كلية ألهندسة = جامعة ألموصل 